\title{
Maternal transport: an opportunity to improve the system of risk- appropriate care
}

\author{
Deborah Burch $\mathbb{1}^{1} \cdot$ Joseph Spinnato ${ }^{2}$
}

Received: 5 November 2020 / Revised: 17 November 2020 / Accepted: 1 December 2020 / Published online: 25 January 2021

This is a U.S. government work and not under copyright protection in the U.S.; foreign copyright protection may apply 2021

In the current edition of The Journal of Perinatology, DeSisto, Oza-Frank, Goodman, Conrey, and Shellhaas [1] report maternal mortality for a 6-year interval in Ohio with a focus upon maternal transport as a critical component in the provision of risk-appropriate maternal care. The authors identified a dearth of published analyses on maternal transport and explored the relationship of maternal transport to pregnancy related deaths. Among 136 pregnancy-related deaths, 15 deaths identified as potentially preventable by transfer to a higher level of care, and only 5 were transported between hospitals. Contributing factors included inadequate response by Emergency Medical Services and lack of transport to a higher level of care. The authors call for improvements to the system of risk-appropriate maternal care, better understanding of local systems' perinatal care capabilities, and reassessment of existing hospital and EMS protocols for caring for and transporting pregnant or postpartum women. Although the current study is from Ohio, the authors suggest that the outcomes observed are likely to be similar across the country.

There are two types of maternal transport studied in this report: (1) EMS transport from the field (home or workplace), and (2) maternal transport from one hospital to another. The issues of and avenues for improvement for each type of transport overlap only minimally, and require separate evaluation and recommendations. With regard to EMS transport of pregnant women from the field, except in clearly life-threatening emergencies (where depending upon the type of emergency transport would be to the closest

Deborah Burch

Deborah.Burch@ flhealth.gov

1 Maternal and Child Health, Division of Community Health Promotion, Florida Department of Health, 2585 Merchant's Row Blvd., Tallahassee, FL 32399, USA

2 Affiliated Clinical Faculty, Division of Maternal Fetal Medicine, Department of Obstetrics and Gynecology, University of South Florida, College of Medicine, Tampa, FL, USA hospital or closest hospital providing obstetric care), EMS transports patients to the planned delivery hospital. These issues seem relatively straight-forward, but require standardization and appropriate training of EMS personnel.

Between hospitals maternal transport is more multifaceted. The hospital to hospital maternal transport during pregnancy for either maternal or fetal benefit is a critically important decision. Accurate and honest assessment of (1) the maternal or fetal condition, (2) the capabilities of the local hospital, and (3) the safety and efficiency of maternal transport versus delivery with post-delivery transport of either mother and/or newborn, are highly complex processes. Ideally, they are done without concern for the financial ramifications to either the transferring physician or hospital.

The transport needs of a mother/fetus dyad are different from postpartum patients' needs and the requirements for facilities that can care for a postpartum patient, particularly late postpartum patients, are different. The late onset maternal deaths are, of course, important to the delivering care providers, but their expertise frequently is not relevant to the patients' medical needs. It is critical to match the needs of the patient to the facility's level of care.

Establishing maternal levels of care is just one piece of the puzzle. In order to make a positive impact, risk appropriate maternal care must be coordinated so that services are accessible and timely [2]. The concept of maternal transport includes transport from home to facility or transport between facilities. Included in the ACOG/SMFMS guidance is the need for regional facilities to collaboratively develop maternal transport plans that foster accurate assessment of risk to ensure women are receiving care at the appropriate level facility [3]. This process entails communication and collaboration with local EMS when transporting from home to hospital and among providers and facilities when there is identified need to transport to a higher level of maternal care.

Maternal health needs can be complex in that they encompass the spectrum from pregnancy through postpartum and 
may include direct obstetric events or other adverse physiological events exacerbated by a pregnancy. Access to risk appropriate maternal care is especially challenging for women living in rural communities [4]. Increasing numbers of rural communities demonstrate a decline or complete loss of obstetric services requiring women to travel long distances to access maternal care. In 2018, the March of Dimes reported that over five million women live in what they term "maternity care desserts" with no providers or facilities offering obstetric services and 10 million women live in counties with limited obstetric access [5]. In addition, there are persistent racial and ethnic disparities with black women being three to four times more likely to experience a pregnancy-related death [2].

There are limitations of the current study particularly the evolving status of adopting standardized levels of maternal care across the nation which includes coordinated systems for maternal transport. However, this study plants a seed that needs to be nurtured. Continued and enhanced funding of the investigatory efforts of state and local maternal mortality committees are critical to the accurate assessment of the adequacy of care when untoward events occur. The findings of this study should compel others to further exploration to identify the barriers and optimize the availability of risk appropriate maternal care for all.

\section{Compliance with ethical standards}

Conflict of interest The authors declare that they have no conflict of interest.

Publisher's note Springer Nature remains neutral with regard to jurisdictional claims in published maps and institutional affiliations.

\section{References}

1. DeSisto C, Oza-Frank R, Goodman D, Conrey E, Shellhaas C Maternal transport: an opportunity to improve the system of riskappropriate care. J Perinatol.

2. Collier AY, Molina RL. Maternal mortality in the United States: updates on trends, causes, and solutions. Neoreviews 2019;20: e561-e574. https://doi.org/10.1542/neo.20-10-e561. PMID: 31575778; PMCID: PMC7377107.

3. American College of Obstetricians and Gynecologists, Society for Maternal-Fetal Medicine, Kilpatrick SJ, Menard MK, Zahn CM, Callaghan WM. Obstetric care consensus \#9: levels of maternal care: (Replaces Obstetric Care Consensus Number 2, February 2015). Am J Obstet Gynecol. 2019;221:B19-B30.

4. Rice-Simpson K. Ongoing crisis in lack of maternity services in rural America. Am J Matern/Child Nurs. 2020;45:132. https://doi. org/10.1097/NMC.0000000000000605.

5. March of Dimes (2018). Nowhere to go: maternity care desserts across the U.S. White Plains, NY. March of Dimes. 